\title{
Un "selfie" in dialisi: valutazione delle abitudini personali degli infermieri di un centro dialisi
}

\author{
Alessandro Capitanini \\ SOC Nefrologia e Dialisi Ospedale San Jacopo, Pistoia, e ASL Toscana Centro, Pistoia - Italy
}

\begin{abstract}
A "Selfie" In Dialysis: Evaluation of Nurses' Personal Habits in a Dialysis Center
Introduction: Healthcare workers represent a population that is well analysed by health studies: since 1976, the Nurses' Health Study has been examining American nurses for health-related risks and is currently recruiting its third cohort. The survey models used are predominantly biomedical, i.e. based on the disease-healing scheme which focuses on purely biological factors with little or no assessment of psychological, behavioural and environmental aspects. The biopsychosocial assessment model, in its multifaceted nature, is probably more suitable for assessing occupational distress as a progressive cause of health worker burnout. It attributes the outcome of illness, as well as that of health, to the numerous, complex interactions of biological, psychological and social factors. In this work, we decided to evaluate the lifestyle and habits of a homogeneous population of nurses, all belonging to an Operative Unit of Nephrology (Pistoia).

Methods: We decided to use unconventional instruments: a "selfie" questionnaire on habits, constructed with scientifically validated items, aimed at the self-assessment of habits, scientifically recognised as determinants of health (nutritional, behavioural, relational, physiological...), correlating it with the analysis of the receipts of the weekly shopping of the family unit of the nurse in the study, according to the principle that "we eat what we buy". Results and conclusion: Nurses evaluated in the study showed a frequent unhealthy lifestyle which can have negative effects on their health, on their family and, consequently, on their work environment. Questionnaire and focus group discussions were appreciated and potentially useful and effective in changing bad habits.
\end{abstract}

Keywords: Burnout, Habits, Hemodialysis, Lifestyle, Nurse, Nutrition

\section{Introduzione}

Il personale sanitario rappresenta una popolazione ben analizzata dagli studi sulla salute: dal 1976, il "Nurses' Health Study" esamina gli infermieri americani per i rischi relativi alla salute (1) e sta, attualmente, reclutando la sua terza coorte.

I modelli di indagine utilizzati sono in prevalenza di tipo biomedico, vale a dire basati sullo schema malattiaguarigione, che si concentra su fattori puramente biologici con scarsa o nulla valutazione degli aspetti psicologici, comportamentali e ambientali.

II modello di valutazione biopsicosociale (2) rappresenta, invece, una strategia di approccio alla persona e attribuisce

Received: July 29, 2021

Accepted: July 30, 2021

Published online: September 15, 2021

Indirizzo per la corrispondenza:

Alessandro Capitanini

SOC Nefrologia Pistoia

Via Ciliegiole 97

51100 Pistoia - Italy

drcapitanini@gmail.com il risultato della malattia, così come quello della salute, alle numerose e complesse interazioni di fattori biologici (genetici, biochimici, nutrizionali, attività fisica...), psicologici (umore, gestione delle emozioni, personalità, comportamento, qualità delle relazioni ecc.) e sociali (culturali, familiari, socioeconomici, lavorativi). Quest'ultimo approccio, nella sua poliedricità, risulta probabilmente più idoneo nella valutazione del disagio lavorativo, in quanto causa progressiva del burnout dell'operatore sanitario, problematica importante e sempre più emergente, amplificata dalla pandemia in atto, che si riflette non solo sulla salute degli operatori ma anche sulla cura e sulla sicurezza dei pazienti (3).

È dagli inizi degli anni '80 che la ricerca in psicologia clinica e sociale affronta il tema del "Burnout", letteralmente traducibile con il termine "bruciarsi" e "dissolversi" e riferito alle "performance" in ambiente lavorativo degli individui. Il burnout è stato inizialmente concettualizzato come un potenziale pericolo soprattutto per gli operatori sanitari e i lavoratori delle risorse umane, divenendo presto un argomento tabù e un problema spesso negato poiché sinonimo, nella mentalità comune, di "non professionalità". Una sorta di "depressione professionale", che si manifesta con spossatezza, perdita di energia (o depersonalizzazione) e declino 
nell'efficacia professionale. Fattori situazionali e ambientali, così come le relazioni interpersonali e sociali, hanno un ruolo cruciale per la comprensione del fenomeno, alla pari delle differenze individuali o di personalità. Un'interessante chiave di lettura del burnout suggerisce di prendere in considerazione il work-life balance, vale a dire l'equilibrio tra vita privata e lavorativa, facendo sì che la domanda essenziale, in caso di burnout, non sia "è dovuto alla persona o al lavoro?", ma, piuttosto, "come può tale fenomeno derivare dall'interazione persona-lavoro?".

L'infermiere della dialisi, in particolare, svolge un lavoro molto specifico e impegnativo: è responsabile non solo della gestione tecnica del rene artificiale e della complessa gestione clinica del malato sottoposto al trattamento dialitico ma ha anche il compito fondamentale di curare la sfera psico-relazionale del paziente. Il paziente dializzato è un malato molto complesso con cui l'infermiere della dialisi trascorre molte ore a settimana. La relazione terapeutica che si crea non è affatto semplice ed è frequente che l'infermiere sperimenti sentimenti di impotenza e, talvolta, di incompetenza.

In questo lavoro, abbiamo deciso di valutare stile di vita e abitudini di una popolazione omogenea di infermieri, tutti appartenenti a un'Unità Operativa di Nefrologia (Pistoia).

Abbiamo deciso di utilizzare strumenti non convenzionali: un questionario, che abbiamo chiamato "selfie", sulle abitudini (Fig. 1), costruito con item scientificamente validati

\begin{tabular}{|c|l|}
\hline \multicolumn{2}{|l|}{ Questionario "selfie" di valutazione delle proprie abitudini } \\
\hline 1 & $\begin{array}{l}\text { Fai attività fisica aerobica almeno } 150 \text { minuti a settimana? } \\
(20)\end{array}$ \\
\hline 2 & Fai esercizi fisici per la forza? (20) \\
\hline 3 & Fai attività fisica > 5 giorni alla settimana? $(20)$ \\
\hline 4 & Sei normopeso, è variato il peso nella tua vita? $(9,10)$ \\
\hline 5 & Fumi? (11) \\
\hline 6 & Bevi meno di due porzioni di alcol al giorno? (12, 13) \\
\hline 7 & Dormi almeno 7 ore a notte? (15) \\
\hline 8 & Alvo quotidiano e regolare? (19) \\
\hline 9 & Ti concedi qualche momento di silenzio e/o riflessione? (20) \\
\hline 10 & Hai prospettive e sei ottimista sul futuro? (20) \\
\hline 11 & Hai una buona vita interiore? (20) \\
\hline 12 & $\begin{array}{l}\text { Eviti cibi raffinati, per esempio quelli che contengono farine 0 } \\
\text { e 00 tipo corn flakes, pane bianco, biscotti, merendine? (21) }\end{array}$ \\
\hline 13 & $\begin{array}{l}\text { Mangi anche piccole quantità di legumi (fagioli, lenticchie, } \\
\text { ceci) e cereali integrali (pasta integrale, riso integrale, farro, } \\
\text { segale, miglio)? (22) }\end{array}$ \\
\hline 14 & Mangi frutta e verdura ad ogni pasto? (22) \\
\hline 15 & $\begin{array}{l}\text { Mangi molto cibo animale, soprattutto carne rossa e affet- } \\
\text { tati? (23) }\end{array}$ \\
\hline 16 & $\begin{array}{l}\text { La prima colazione è un pasto ricco e vario o mangi caffè e } \\
\text { cornetto? (24) }\end{array}$ \\
\hline
\end{tabular}

Fig. 1 - Questionario ("selfie") di valutazione delle proprie abitudini.
(4-24), mirato all'autovalutazione delle abitudini, riconosciute scientificamente come determinanti per la salute (nutrizionali, comportamentali, relazionali, fisiologiche ...), correlandolo, poi, con l'analisi degli scontrini della spesa settimanale del nucleo familiare di appartenenza dell'infermiere in studio, secondo il principio per cui "mangiamo quello che compriamo" (5). II personale ha aderito con interesse e curiosità al progetto proposto.

\section{Materiali e metodi}

La popolazione studiata era costituita da dipendenti della ASL Area Vasta Toscana Centro, in particolare dagli infermieri di un reparto di Nefrologia. Sono stati arruolati tutti e 37 gli infermieri della SOC di Nefrologia di Pistoia, di cui 32 donne e 5 uomini. L'indagine è stata realizzata in epoca pre-Covid19. Tutti i partecipanti erano di età superiore ai 18 anni e avevano un'anamnesi medica negativa per patologie significative, a eccezione, per esempio, di occasionali dolori alla schiena o di ipertensione arteriosa. Non è stata proposta alcuna ricompensa economica ma a tutti i partecipanti è stato offerto di ricevere i loro risultati individuali dopo la fine dello studio.

\section{Scontrini della spesa}

Abbiamo analizzato le abitudini alimentari del nostro personale, chiedendo a tutti i partecipanti di raccogliere accuratamente tutti gli scontrini della spesa di una settimana. Dall'analisi degli scontrini siamo risaliti ai cibi acquistati. Tutte le bevande non nutrizionali (acqua pura e tè non zuccherato) sono state trascurate per motivi di conformità e praticità. Gli alimenti sono stati categorizzati seguendo le Linee Guida dell'Healthy Eating Plate (4), detto “piatto Harvard", e le quantità sono state valutate percentualmente al totale.

\section{Classificazione dei cibi (4)}

- Ortaggi

- Frutta

- Cereali integrali

- Cereali raffinati (farine 0 e 00)

- Proteine sane (pesce, pollame, uovo, legumi, semi oleaginosi).

- Carne rossa, salumi, carni conservate

- Latticini

- Junk food (torta, caramelle, gelati, merendine, cioccolato con cacao $<70 \%$, bevande zuccherate)

\section{Questionario "selfie"}

Abbiamo creato un questionario (Fig. 1) inserendo valutazioni su abitudini fisiologiche, sfera comportamentale e sfera 
emotiva e fisiologica, utilizzando item validati dalla letteratura (5-24). Abbiamo esplorato l'attività fisica $(7,8)$, il peso corporeo e le sue modificazioni nel tempo $(9,10)$, la quantità di sonno (15), le abitudini alimentari e voluttuarie (11-13) e alcuni aspetti della sfera emozionale (20).

\section{Risultati}

Sono stati studiati 37 infermieri della Nefrologia di Pistoia, 32 femmine e 5 maschi, età media $43 \pm 10$ anni. Le risposte al questionario hanno dato i seguenti esiti:

attività fisica: il 70\% è sedentario e non raggiunge l'obiettivo minimo stabilito dall'OMS di 150 minuti di attività aerobica settimanale per adulti sani. L'85\% degli infermieri non esegue esercizi fisici per la forza muscolare. Solo il $16,5 \%$ degli infermieri pratica attività fisica quotidiana (5 volte a settimana o più);

peso corporeo: il $67 \%$ del campione è in sovrappeso e ha avuto variazioni di peso, in aumento (escluse le gravidanze), nel corso della vita. In particolare tutti hanno incrementato il peso corporeo rispetto a quello intorno ai 18 anni;

funzioni fisiologiche: il $44 \%$ degli infermieri non raggiunge le 7 ore di sonno quotidiane e il $32 \%$ non ha una buona regolarità intestinale, con prevalenza di stitichezza;

abitudini voluttuarie: i fumatori rappresentano il $25 \%$ del campione (in media 12 sigarette al giorno) e fanno uso di alcolici (non superalcolici), con superamento delle 2 porzioni nel $38 \%$ dei casi;

sfera emozionale: il $32 \%$ del campione non ha, nella giornata, momenti di silenzio e di riflessione. II $46 \%$ vede il futuro con pessimismo e incertezza;

alimentazione: il 55\% del campione usa cibi raffinati/ processati. L'11\% usa regolarmente junk food. II $60 \%$ non mangia abitualmente legumi e cereali integrali.

Frutta e verdura non sono consumate a tutti i pasti dal $20 \%$ dei soggetti. II $33 \%$ usa molto cibo animale. II $48 \%$ fa una prima colazione insufficiente.

\section{Scontrini della spesa}

La spesa settimanale viene considerata nel "piatto Pistoia" confrontato con il "piatto Harvard". Fuori dal piatto Harvard e, quindi, genericamente da considerare negativamente sono risultati: $15 \%$ di junk food, $20 \%$ di cereali raffinati e $20 \%$ di carne rossa e latticini.

Corrispondenti ai cibi "sani": ortaggi e frutta $30 \%$, cereali integrali $1 \%$ e proteine sane $14 \%$.

II confronto con il piatto Harvard si evidenzia nella Figura 2.

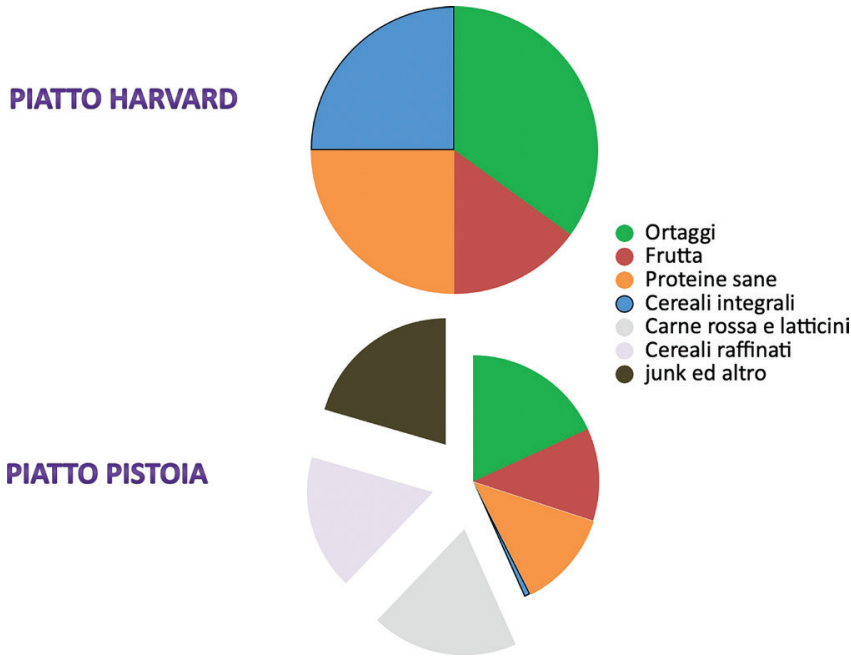

Fig. 2 - Confronto fra il "piatto" del mangiar sano proposto da Harvard e il "piatto" degli infermieri della dialisi di Pistoia ricostruito in base agli alimenti acquistati in una settimana.

\section{Discussione}

La commissione europea e l'organizzazione mondiale della sanità ripetono, come un mantra, che uno stile di vita sano è la chiave per il nostro benessere e un antidoto a molte malattie (l'80\% delle patologie cardiovascolari potrebbe, infatti, essere evitato con un sano stile di vita, secondo l'Istituto Superiore di Sanità). Tutti condividiamo l'idea secondo cui la prevenzione è parte integrante delle azioni a tutela della nostra salute, ma, talvolta, non c'è molta chiarezza su cosa significhi prevenire. Nonostante il loro livello educativo e l'occupazione in ambito sanitario, gli infermieri valutati nello studio hanno dimostrato di avere uno stile di vita, non sempre corretto, che può avere effetti negativi sulla loro salute e, conseguentemente, sulla loro sfera familiare e lavorativa. Sono stati osservati un'alta percentuale di pratiche alimentari inappropriate, di sedentarietà e di sovrappeso e modelli di sonno inadeguati.

Giova ricordare quanto le abitudini, come, per esempio, la sedentarietà (25), possano influire negativamente sul rendimento lavorativo e sulle relazioni interpersonali ed essere propedeutiche ad altre cattive abitudini, come fumare e abusare di alcolici e del cibo.

II confronto con il gruppo (focus group) (26), che è seguito alla discussione dei risultati, ha permesso a molti di prendere consapevolezza su abitudini consolidate ma scorrette e di avere una spinta al cambiamento, che, in molti, hanno adottato.

Perseguire uno stile di vita sano presuppone soprattutto una conoscenza puntuale di cosa ci faccia realmente bene e di che cosa sia da considerare davvero "sano". Dobbiamo, dunque, prendere in considerazione l'idea che la salute richieda maggiore consapevolezza. 


\section{Conclusioni}

Il questionario "selfie" ha rappresentato uno strumento di riflessione e di consapevolezza. In particolare, la spiegazione dei vari item del test, prima di averlo compilato e dopo, ha chiarito concetti ed evidenze scientifiche spesso ignorati.

Le abitudini rappresentano un rifugio dell'essere umano perché permettono, come un pilota automatico, di vivere senza sforzi organizzativi. Purtroppo, però, se sono "cattive" abitudini, inducono a scelte sbagliate, che si ripercuotono sul lavoro e, più in generale, sulla salute delle persone. L'associazione, adottata in questo studio, di un questionario self report ("selfie") alla discussione in gruppo dei risultati, avvenuta con la tecnica del focus group (26), si è rivelata utile e proficua. Affrontare il burnout non può prescindere da un'accurata analisi del worklife balance, perché spesso i problemi non nascono solo, o comunque non esclusivamente, sul lavoro.

\section{Disclosures}

Conflict of interest: The authors declare no conflict of interest. Financial support: This research received no specific grant from any funding agency in the public, commercial, or not-for-profit sectors.

\section{Bibliografia}

1. Belanoger, Charlene; Speizer, Frank E.; Hennekens, Charles H.; Rosner, Barnard; Willett, Walter; Bain, Chritopher. The Nurses' Health Study. American Journal of Nursing 1980;80(7): 1333. CrossRef

2. Engel GL. The need for a new medical model: a challenge for biomedicine. Science. 1977;196(4286):129-136. CrossRef PubMed

3. Feskanich D, Hastrup JL, Marshall JR, et al. Stress and suicide in the Nurses' Health Study. J Epidemiol Community Health. 2002;56(2):95-98. CrossRef PubMed

4. The Healthy Eating Plate, please see The Nutrition Source, Department of Nutrition, Harvard T.H. Chan School of Public Health, Online, and Harvard Health Publications, Online.

5. Willett WC, Sampson L, Browne ML, et al. The use of a selfadministered questionnaire to assess diet four years in the past. Am J Epidemiol. 1988;127(1):188-199. CrossRef PubMed

6. Martínez de Victoria Muñoz E. Do we know what we eat? A nutrition perspective. Nutr Hosp. 2018 Jun 12;35(Spec No4):61-65. CrossRef PubMed

7. Manson JE, Hu FB, Rich-Edwards JW, et al. A prospective study of walking as compared with vigorous exercise in the prevention of coronary heart disease in women. $N$ Engl J Med. 1999;341(9):650-658. CrossRef PubMed

8. Piercy KL, Troiano RP, Ballard RM, et al. The Physical Activity Guidelines for Americans. JAMA. 2018;320(19):2020-2028. CrossRef PubMed

9. Romieu I, Willett WC, Stampfer MJ, et al. Energy intake and other determinants of relative weight. Am J Clin Nutr. 1988; 47(3):406-412. CrossRef PubMed
10. Fine JT, Colditz GA, Coakley EH, et al. A prospective study of weight change and health-related quality of life in women. JAMA. 1999;282(22):2136-2142. CrossRef PubMed

11. Colditz GA, Bonita R, Stampfer MJ, et al. Cigarette smoking and risk of stroke in middle-aged women. $N$ Engl J Med. 1988;318(15):937-941. CrossRef PubMed

12. Xi B, Veeranki SP, Zhao M, Ma C, Yan Y, Mi J. Relationship of Alcohol Consumption to All-Cause, Cardiovascular, and Cancer-Related Mortality in U.S. Adults. J Am Coll Cardiol. 2017;70(8):913-922. CrossRef. PubMed. CrossRef. Google Scholar. PubMed

13. Colditz GA. A prospective assessment of moderate alcohol intake and major chronic diseases. Ann Epidemiol. 1990;1(2): 167-177. CrossRef PubMed

14. Hu FB, Stampfer MJ, Manson JE, et al. Dietary saturated fats and their food sources in relation to the risk of coronary heart disease in women. Am J Clin Nutr. 1999;70(6):1001-1008. CrossRef PubMed

15. Liu Y, Wheaton AG, Chapman DP, Cunningham TJ, Lu H, Croft JB. Prevalence of Healthy Sleep Duration among Adults-united States, 2014. MMWR Morb Mortal Wkly Rep. 2016;65(6): 137-141. CrossRef PubMed

16. Liu S, Willett WC, Manson JE, Hu FB, Rosner B, Colditz G. Relation between changes in intakes of dietary fiber and grain products and changes in weight and development of obesity among middle-aged women. Am J Clin Nutr. 2003;78(5): 920-927. CrossRef PubMed

17. Hu FB. Plant-based foods and prevention of cardiovascular disease: an overview. Am J Clin Nutr. 2003;78(3)(suppl): 544S-551S. CrossRef. Review. PubMed

18. Kraemer WJ, Adams K, Cafarelli E, et al; American College of Sports Medicine. American College of Sports Medicine position stand. Progression models in resistance training for healthy adults. Med Sci Sports Exerc. 2002;34(2):364-380. CrossRef PubMed

19. Sharma A, Rao S. Constipation: Pathophysiology and Current Therapeutic Approaches. Handb Exp Pharmacol. 2017;239: 59-74. CrossRef PubMed

20. Philips KH, Brintz CE, Moss K, Gaylord SA. Didgeridoo Sound Meditation for Stress Reduction and Mood Enhancement in Undergraduates: A Randomized Controlled Trial. Glob Adv Health Med. 2019;8:1-10. CrossRef PubMed

21. Schulte EM, Avena NM, Gearhardt AN. Which foods may be addictive? The roles of processing, fat content, and glycemic load. PLoS One. 2015;10(2):e0117959. CrossRef PubMed

22. Serafini M, Pelusol. Functional Foods for Health:The Interrelated Antioxidant and Anti-Inflammatory Role of Fruits, Vegetables, Herbs, Spices and Cocoa in Humans. Curr Pharm Des. 2016; 22(44):6701-6715. CrossRef PubMed

23. Wolk A. Potential health hazards of eating red meat. J Intern Med. 2017;281(2):106-122. CrossRef PubMed

24. Uzhova I, Fuster V, Fernández-Ortiz A, et al. The Importance of Breakfast in Atherosclerosis Disease: Insights From the PESA Study. J Am Coll Cardiol. 2017;70(15):1833-1842. CrossRef PubMed

25. Colombo E, Senn L. I costi economici e sociali della sedentarietà. Med Sport (Roma). 2015;68:345-356.

26. Traynor M. Focus group research. Nurs Stand. 2015;29(37): 44-48. CrossRef PubMed 